\title{
Analysis of pre-edge XANES spectra of Fe:SiO4 system by using machine learning methods
}

\author{
D. Pashkov ${ }^{1,2}$, A.A. Guda1 , S.A. Guda ${ }^{2}$, A.V. Soldatov ${ }^{1}$
}

${ }^{1}$ The Smart Materials Research Institute, Southern Federal University, Sladkova 178/24, 344090 Rostov-on-Don, Russia , ${ }^{2}$ Institute of mathematics, mechanics and computer science, Southern Federal University, Milchakova 8a, 344090 Rostov-on-Don, Russia

$$
\text { pashkov@sfedu.ru }
$$

Advanced quantitative analysis of the local atomic geometry around active catalytic sites requires novel experimental method e.g. the pre-edge structure of X-ray absorption near edge spectra (XANES) measured in the high-energy resolution fluorescence detected mode, the so-called HERFD-XANES. However, there is no widely used ab initio theoretical method which could be routinely applied to the analysis of such experimental data except parametric multiplet calculations. To overcome the procedure of adjusting of parameters is the using of local DFT Hamiltonian constructed on the basis of Wannier orbitals - the so called multiplet ligand-field theory (MLFT) [1]. Pre-edge region of X-ray absorption spectra could be calculated using the XTLS code in the framework of multiplet ligand-field theory using maximally localized Wannier functions (MLWF).

Computation of pre-edge XANES spectra according to MLFT approach is a complicated process, which requires using a lot of software, such as: Wien2k, Wannier90, XTLS code and some additional programs and scripts. We developed «W2auto» program, which automates all process of pre-edge XANES computation. «w2auto» emulates work in w2web interface of Wien2k software and provides opportunity to run all necessary programs without user access.

In recent years machine learning has become a powerful instrument for solving scientific problems. In this work we have applied machine learning methods for analysis of the $\mathrm{Fe}: \mathrm{SiO}_{4}$ pre-edge XANES spectra. As recently shown, machine learning methods have been successfully applied to the quantitative analysis of spectroscopic data in general and of X-ray near edge spectroscopy (XANES) in particular [2]. In the present work we show applicability of machine learning methods to retrieve structural information in system $\mathrm{Fe}: \mathrm{SiO}_{4}$. In this research we have collected 60 pre-edge XANES spectra in differrent coordination (from 2-fold to 6-fold) (Fig. 1) and oxidation states $\left(\mathrm{Fe}^{2+}\right.$ and $\left.\mathrm{Fe}^{3+}\right)$ using «w2auto» program. Fig. 2 shows the scatter plot of spectra made by expected values of absorption intensity and integral under spectrum.

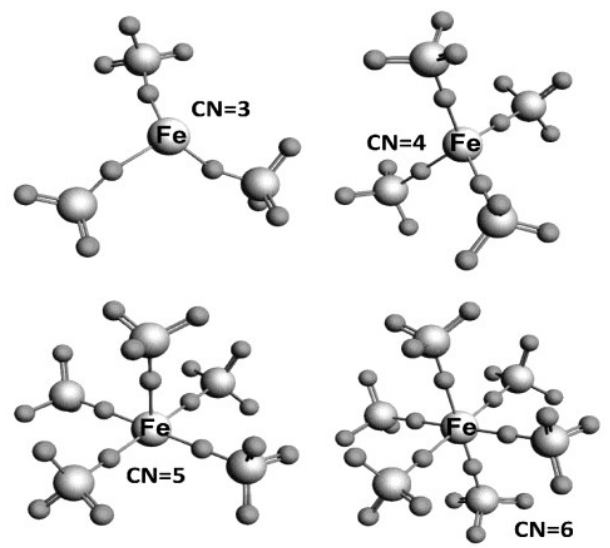

Figure 1. Fe:SiO4 in different coordinations

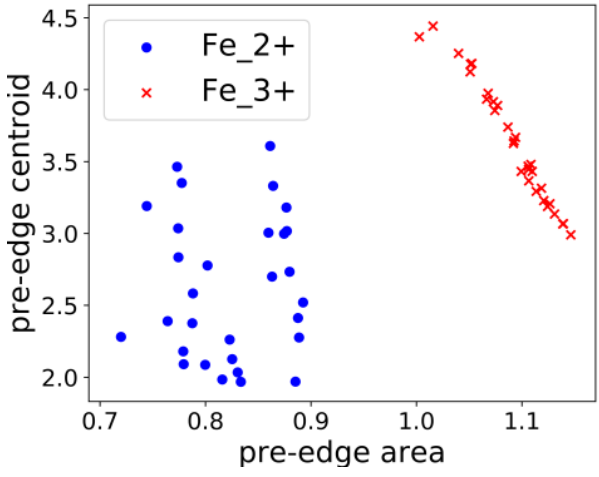

Figure 2. Scatter plot of training dataset for different oxidation states of Fe.

We used this dataset to train and validate several machine learning methods (Decision Tree, ExtraTrees, SVM, Logistic regression and neural network) to determine both coordination number and oxidation state by spectrum.

The work was supported by grant of President of Russia for young scientists (MK-2730.2019.2).

[1] Gorelov E., Guda A.A., Soldatov M.A., Guda S.A., Pashkov D., Tanaka A., Lafuerza S., Lamberti C., Soldatov A.V. (2018). Radiation Physics and Chemistry 108105. MLFT approach with p-d hybridization for ab initio simulations of the pre-edge XANES.

[2] Martini A., Guda S.A., Guda A.A., Smolentsev G., Algasov A., Usoltsev O.,et al. (2019). Computer Physics Communications. PyFitit: the software for quantitative analysis of XANES spectra using machine-learning algorithms.

Keywords: XANES, pre-edge, Wannier function, MLFT, machine learning

Acta Cryst. (2021), A77, C477 